\title{
Evaluation of Micronucleus Frequencies in Exfoliated Buccal Cells of Potentially Malignant Disorder Subjects Using Feulgen Stain
}

\author{
Gunjan T. Dave ${ }^{1}$, Neelampari Parikh², Nilesh Patel $^{3}$, Hemal joshi ${ }^{3}$ \\ ${ }^{1}$ Department of Oral Pathology, Gujarat University, Ahmedabad, Gujarat, India, ${ }^{2}$ Department of Oral and Maxillofacial Pathology, \\ Karnavati School of Dentistry, Gandhinagar, Gujarat, India, ${ }^{3}$ Department of Oral and Maxillofacial Pathology, Siddhpur Dental \\ College, Siddhpur, Gujarat, India
}

Email for correspondence: gunjan_dave29@rediffmail.com

\begin{abstract}
Introduction: Oral squamous cell carcinoma generally arises from oral potentially malignant disorders (OPMDs) through a complex process of genetic alterations such as chromosomal alterations, epigenetic alterations, and/or DNA changes. If these lesions are detected in early stages, i.e., during stage of PMDs through specific and sensitive tests, it can markedly improve the survival rates of patients. Micronucleus (MN) is one such suitable and reliable test to detect genotoxic effects of various carcinogens. Aims: The aim of the present study was to evaluate and compare the levels of micronuclei in OPMDs as well as to correlate micronuclei frequency in different grades of dysplasia. Materials and Methods: The study cases were divided into two groups, Group I consisting of thirty healthy controls and Group II of thirty histopathologically proven cases of OPMDs. The exfoliated cells were collected from buccal mucosa/lesional area of both study groups and were stained with Feulgen stain and evaluated under microscope for MN. Statistical analysis was done using ANOVA test and correlation was calculated using Pearson's correlation using SPSS 18.0 software. Results: According to the present study, MN and MN percentage (MN\%) increased from normal to potentially malignant disorder cases with statistically significant difference. A highly positive correlation (0.946) between grades of dysplasia and $\mathrm{MN}$ formation was obtained. Conclusion: The present study concludes that the occurrence of MN and MN\% increases from controls to potentially malignant disorder cases. Among PMDs, the occurrence of $\mathrm{MN}$ and $\mathrm{MN} \%$ was highest in patients of oral submucous fibrosis followed by leukoplakia and lichen planus patients. And finally, with increase in grades of dysplasia, in PMD subjects, the formation of MN increases.
\end{abstract}

Key words: Dysplasia, Feulgen stain, micronucleus, potentially malignant disorders

\section{INTRODUCTION}

Oral squamous cell carcinoma (OSCC) generally arises from oral potentially malignant disorders (OPMDs) such as leukoplakia, oral submucous fibrosis, oral lichen planus, erythroplakia, etc., through a complex process of genetic alterations such as chromosomal alterations, epigenetic alterations,

\section{Quick Response Code Article Info:}

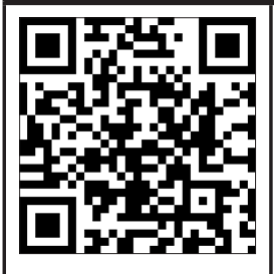

doi: 10.5866/2019.11.10086

Received: $10-07-2019$

Revised: 09-08-2019

Accepted: 16-08-2019

Available Online: 01-10-2019, (www.

nacd.in) ( ) NAD, 2019 - All rights reserved and/or DNA changes. ${ }^{[1]}$ If these lesions are detected in early stages, i.e., during stage of PMDs through specific and sensitive tests, it can markedly improve the survival rates of patients. ${ }^{[2]}$ Application of such simple, sensitive, and specific test seems to require hours for early diagnosis. During the past four decades, the introductions of a large number of relatively rapid genetic tests for assessing the activity of genotoxic agents have been documented. Among them, micronucleus (MN) assessment in exfoliated buccal cells of OPMDs appears to be one of the most suitable and reliable tests to detect the genotoxic effects of various carcinogens. ${ }^{[1]} \mathrm{MN}$ is a small extranuclear particle of DNA formed due to fragmentation of chromosome or chromosomes lag behind which fail to be included in the main nucleus 
of daughter cells. ${ }^{[3]}$ It is visibly round or oval cytoplasmic chromatin mass in the extranuclear vicinity, originated from aberrant mitosis consists of eccentric chromosomes, chromatid fragments or whole chromosomes which failed to reach spindle poles during mitosis. ${ }^{[4]}$

The MN assay in exfoliated cell involves microscopic analysis of exfoliated buccal epithelial cell smears to determine the prevalence of micronucleation, which may be an indicator of numerical or structural chromosomal aberrations. The micronuclei assay in exfoliated cells is a reliable, sensitive, and noninvasive and technically easy test to perform. ${ }^{[5]}$ Increased MN count in OPMDs is well documented in various studies ${ }^{[6]}$ Research in this field is quite promising; hence, this study was conducted to evaluate and compare the levels of micronuclei in OPMDs as well as to correlate micronuclei frequency in different grades of dysplasia in OPMDs using DNA specific Feulgen stain.

\section{MATERIALS AND METHODS}

\section{Patient Selection}

The study was conducted in the Department of Oral and Maxillofacial Pathology and Department of Oral Medicine and Radiology of Karnavati School of Dentistry, Uvarsad, Gandhinagar, the data collection consisted of randomly selected sixty cases and divided into following groups.

Group I: Thirty healthy controls with no oral lesions, no previous tobacco-related habits, and no previous medical history with age and sex matching with diseased cases

Group II: Thirty histopathologically proven cases of OPMDs such as oral leukoplakia $(n=10)$, oral submucous fibrosis $(n=10)$, and oral lichen planus $(n=10)$ [Graph 1 and Figures 1-3].

In each group, cases were given a standard questionnaire interview and their history was recorded. Routine hematological investigations were carried out in the study cases before biopsies.

\section{Collection of Exfoliated cells}

The participants of the study were asked to rinse their mouth with water and a premoistened wooden spatula was used to sample cells from the buccal mucosa of Group I (control) cases and from lesional area of Group II (potentially malignant disorder). The cells were immediately smeared

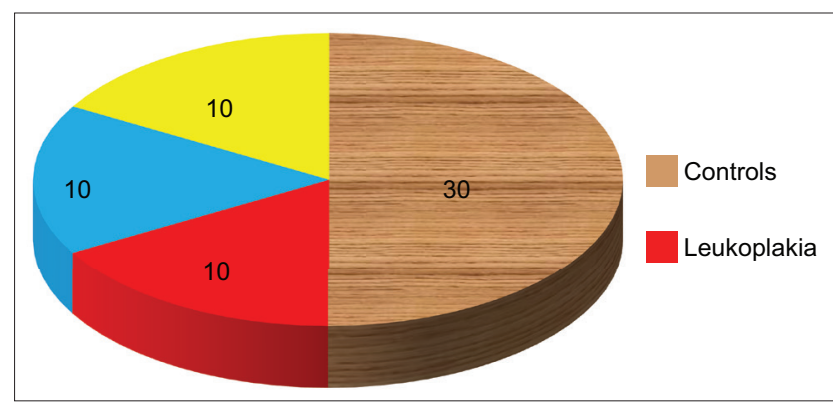

Graph 1: Distribution of total number of cases in the present study

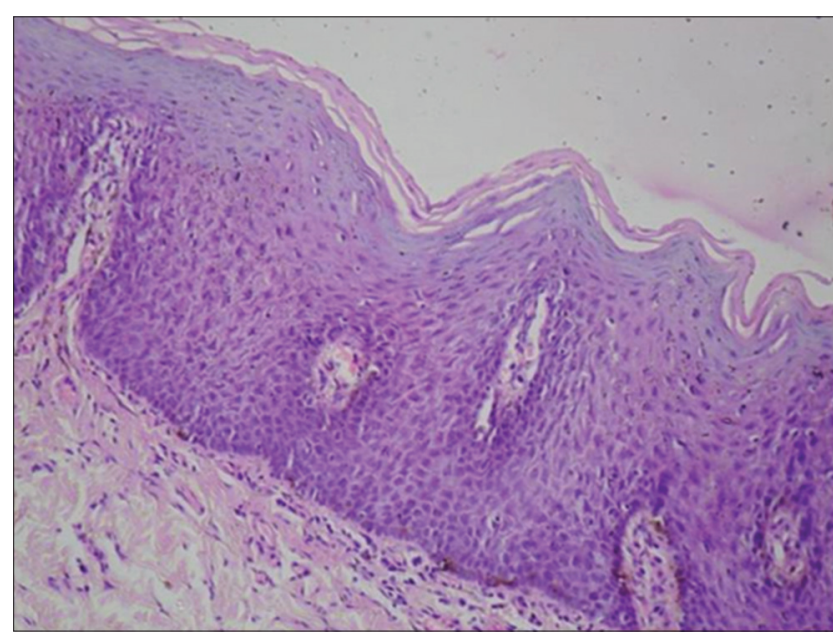

Figure 1: Photomicrograph showing hyperkeratosis ( $\mathrm{H}$ and $\mathrm{E}$ stain, $\times 10$ )

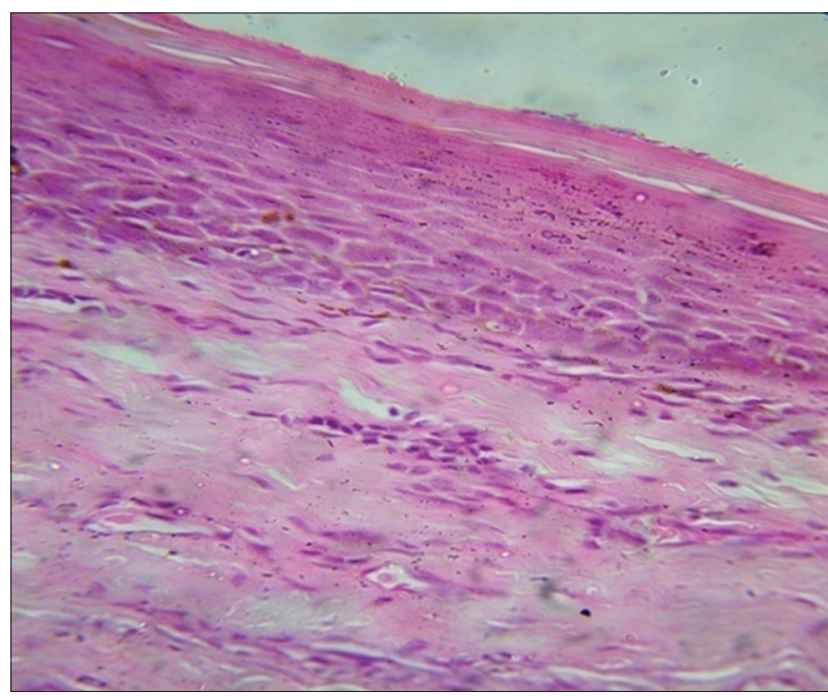

Figure 2: Photomicrograph showing oral submucous fibrosis with atrophic epithelium and area of hyalinization $(\mathrm{H}$ and $\mathrm{E}$ stain, $\times 10$ ) 
on precleaned microscopic slides. The smears were fixed with fixative solution for minimum 15 min $(80 \%$ methyl alcohol + glacial acetic acid fixative mixed in ratio $3: 1$ ) and stained with Feulgen stain. Incisional/punch biopsy in cases with oral lesions was taken. $\mathrm{H}$ and $\mathrm{E}$ stained slides of Group II cases were evaluated for histopathological diagnosis and to determine the degree of epithelial dysplasia.

\section{Evaluation of MN in Cytology}

All the slides of cytology smears were observed under low magnification $(\times 10)$ for screening and high magnification $(\times 40)$ for counting of $\mathrm{MN}$

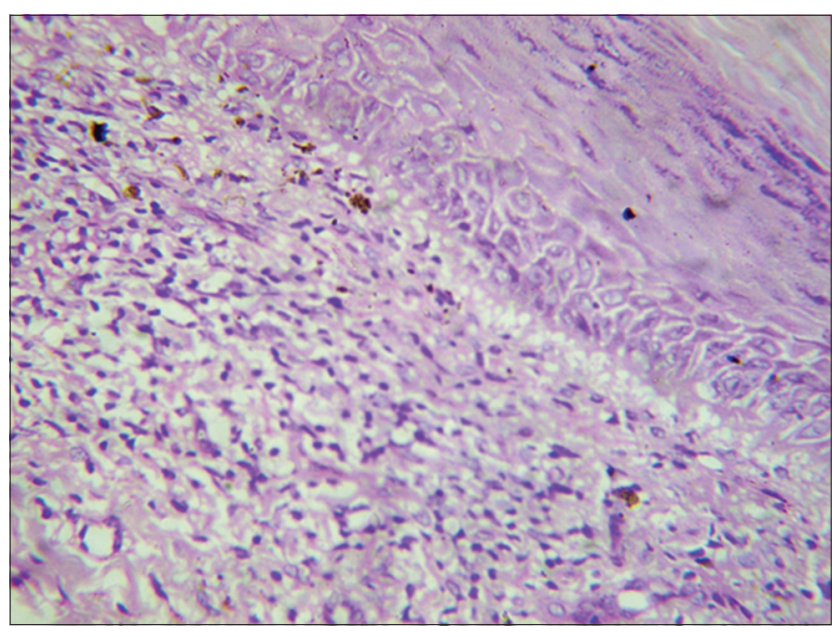

Figure 3: Photomicrograph showing oral lichen planus with basilar cell degeneration, juxtaepithelial lymphocyte infiltration, and melanin incontinence $(\mathrm{H}$ and $\mathrm{E}$ stain, $\times 10)$

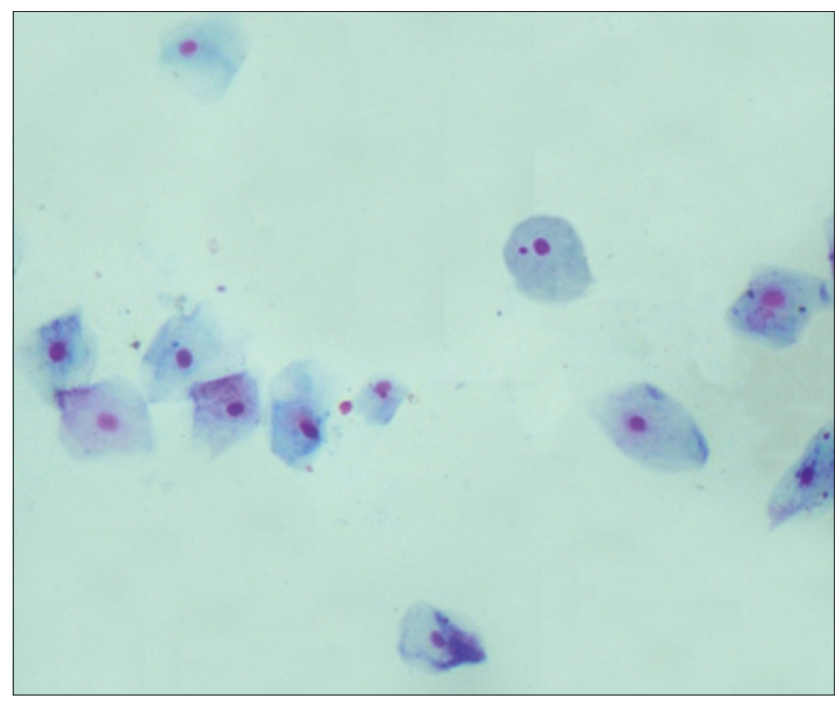

Figure 4: Photograph showing a cytosmear of buccal epithelial cells with single micronucleus (Feulgen staining, $\times 10$ )
[Figures 4 and 5]. The zigzag method was used for screening slides and 500 cells with intact nuclei and cell boundaries were counted per patient.

\section{Scoring Criteria}

The scoring of MN was done according to criteria given by Tolbert et al. (1992):

- Rounded smooth perimeter

- Less than one-third diameter of the associated nucleus, but large enough to discern shape and color

- Staining intensity similar to that of nucleus

- Texture similar to that of nucleus

- Same focal plane as nucleus

- Absence of overlap, with or bridge to the nucleus.

The exclusion criteria considered when scoring of MN was done were as follows: (1) Broken eggs (2) nuclear budding(3) binucleated cell(4)karyorrhexis

(5) karyolysis observations were expressed in form of number of $\mathrm{MN}$ and MN percentage (MN\%) in each patient.

MN\% was counted using this formula: $100 \times$ Total number of micronucleus found in a Group No. of cells counted per patient $(500) \times$ Total number of patients in a Group

\section{Statistical Analysis}

The data obtained were statistically analyzed using ANOVA test and correlation was calculated using Pearson's correlation using SPSS 18.0 software.

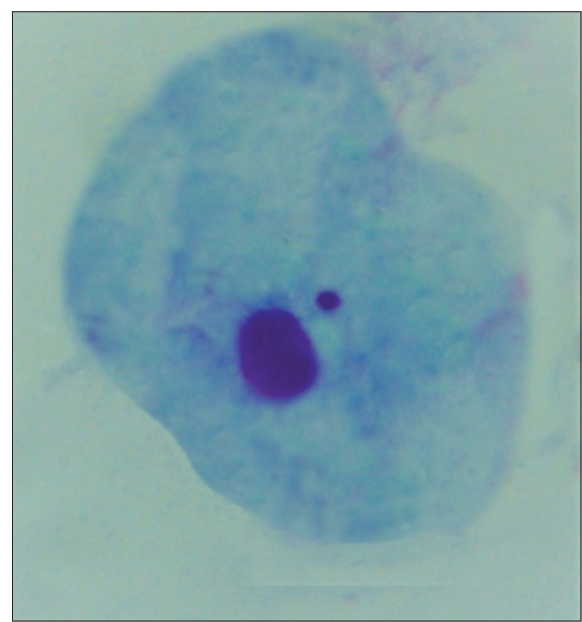

Figure 5: Photograph showing a cytosmear of buccal epithelial cell with single micronucleus (Feulgen staining, $\times 40$ ) 
Table 1: Comparison of mean of MN and MN\% in Group I (controls) and Group II (PMDs)

\begin{tabular}{lccccc} 
Group & No. of cases & Mean of MN+Standard deviation & MN\% & $\boldsymbol{P}$-value (ANOVA) & Statistical significance \\
\hline $\begin{array}{l}\text { Group I } \\
\text { (control cases) }\end{array}$ & 30 & $3.30+1.022$ & 0.66 & 0.001 & Significant \\
$\begin{array}{l}\text { Group II } \\
\text { (PMDs) }\end{array}$ & 30 & $6.47+2.240$ & 1.33 & & \\
\hline
\end{tabular}

PMDs: Potentially malignant disorders

Table 2: Mean of MN in Group II (potentially malignant disorder cases) (leukoplakia, OSMF, and lichen planus)

PMDs

Mean of MN+Standard deviation

Leukoplakia (10 cases) $\quad 7+2.108$

OSMF (10 cases)

$7.80+2.098$

Lichen planus (10 cases)

$4.60+1.075$

PMDs: Potentially malignant disorders, OSMF: Oral submucous fibrosis

Table 3: Comparison of occurrence of MN in Group B PMDs

(leukoplakia, OSMF, and lichen planus) (ANOVA test)

\begin{tabular}{lcl} 
Groups & $\boldsymbol{P}$-value & Significance \\
\hline OSMF versus lichen planus & 0.002 & $\mathrm{~S}$ \\
Lichen planus versus leukoplakia & 0.018 & $\mathrm{~S}$ \\
Leukoplakia versus OSMF & 0.536 & $\mathrm{NS}$ \\
\hline
\end{tabular}

$n=30$. PMDs: Potentially malignant disorders, OSMF: Oral submucous fibrosis

\section{RESULTS}

The study population comprised 60 subjects, as shown in Table 1 and pie chart of which there were 26 males and 4 females in Group I and equal number of males and females in Group II. Mean of MN with standard deviation in Group I and Group II was $3.30+1.022$ and $6.47+2.240$, respectively. MN\% was calculated to be $0.66 \%$ and $1.33 \%$, respectively. The occurrence of $\mathrm{MN}$ and MN\% increases in Group II was almost two folds (or double) as compared to Group I. On comparing the occurrence of MN between Group I and Group II, by applying ANOVA test $P=0.001$ which signifies a statistically significant difference.

As shown in Table 2 and Graph 2, mean of MN and MN\% was found to be least in lichen planus cases $(4.60$ and $0.92 \%$ ) and was highest among oral submucous fibrosis (OSMF) cases (7.80 and 1.68\%), respectively. Table 3 shows $P$ value obtained between Group II cases for the occurrence of MN. There is a statistically significant difference between leukoplakia and lichen planus and between OSMF and lichen planus $(P<0.05)$ for occurrence of MN. There was no statistically significant difference

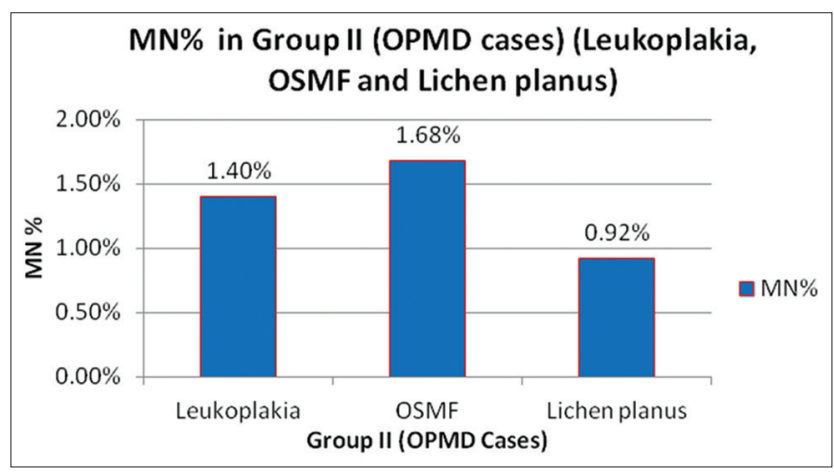

Graph 2: Micronucleus percentage in Group II

between leukoplakia and $\operatorname{OSMF}(P>0.05)$ for occurrence of MN.

Of 10 cases of leukoplakia 3 cases were histopathologically diagnosed as hyperkeratosis with no dysplasia, 2 cases were hyperkeratosis with mild dysplasia, 4 cases were hyperkeratosis with moderate dysplasia, and 1 case was of hyperkeratosis with severe dysplasia.

There were 10 cases of OSMF of which histopathologically 4 showed no dysplasia while other 4 showed mild dysplasia and 2 cases showed moderate dysplasia. Among 10 cases of oral lichen planus, on histopathological examination only 2 cases showed mild dysplasia and remaining 8 cases did not show any features of dysplasia. No cases of carcinoma in situ were found in the present study.

All 30 cases of Group B, as shown in Table 4, there was no dysplasia in 15 cases, mild dysplasia in 8 cases, moderate dysplasia in 6 cases, and severe dysplasia in 1 case.

The mean value of $\mathrm{MN}$ and $\mathrm{MN} \%$ in different grades of dysplasia was found, as shown in Table 2 and Graph 2 respectively. On correlating frequency of MN formation with grades of dysplasia $r=0.946$ was obtained which indicated a highly positive correlation between grades of dysplasia and MN formation (Table 4). 
Table 4: Frequency of MN in different grades of dysplasia in Group II (potentially malignant disorder cases)

\begin{tabular}{|c|c|c|c|c|c|}
\hline Grades of dysplasia & No. of cases (of 30$)(\%)$ & Mean MN value+Standard deviation & MN\% & r-value & Correlation \\
\hline No dysplasia & $15(50)$ & $5.86+2.294$ & 1.18 & 0.946 & Positive correlation \\
\hline Mild dysplasia & $8(26.67)$ & $6.12+1.807$ & 1.25 & & \\
\hline Severe dysplasia & $1(3.33)$ & 9 & 1.80 & & \\
\hline
\end{tabular}

* No cases of carcinoma in situ were found in the present study. r-value $=0 \rightarrow$ No correlation; $r$-value $=+1 \rightarrow$ Positive correlation $(0.946 \sim 1)$; r-value $=1 \rightarrow$ Negative correlation

\section{DISCUSSION}

Screening the individuals who are at high risk of malignancy development is more decisive in preventing and reducing the mortality rate than the costly and painful treatment. ${ }^{[7]}$ An early diagnostic test, such as MN test, would be highly beneficial to check the progress of PMDs to squamous cell carcinoma followed by its early treatment. ${ }^{[8]} \mathrm{MN}$ scoring can be used as a biomarker to identify different PMDs much earlier than the manifestations of clinical features and might specifically be exploited in the screening of highrisk population. ${ }^{[9]}$

Micronuclei can be used as a diagnostic tool, to screen individuals having PMDs, and know whether they are at risk of transformation to malignancy. ${ }^{[10]}$ Hence, the present study was performed with aim of evaluating MN frequencies in PMDs using Feulgen stain. Furthermore, frequencies of MN in different grades of dysplasia of these PMDs were evaluated.

Tobacco, particularly betel nut chewing, is one of the main causative agents of PMDs. ${ }^{[1]}$ The present study, being conducted in Gujarat state, patients who presented with PMDs had consumed tobacco, which was mainly in the form of chewing or smoking. The N-nitrosamine content of tobacco (betel quid) being carcinogenic in nature causes chromosomal aberration and leads to formation of micronuclei in oral epithelial cells. ${ }^{[11]}$

In the present study, exfoliated buccal cells were used for MN detection; this can be arguably explained on three bases. First, as buccal mucosa provides a barrier to potential carcinogens that can be metabolized to generate potential reactive products, approximately $90 \%$ of human cancers originate from these epithelial cells. They represent a preferred target site for early genotoxic events induced by carcinogenic agents entering the body by way of inhalation and ingestion. ${ }^{[12]}$ Second, the collection of buccal cells is arguably the least invasive method available for measuring DNA damage in humans, especially in comparison to obtaining blood samples or tissue biopsies, as it is the most accessible site in the oral cavity, and its covering epithelium is non-keratinized. ${ }^{[13,14]}$ Third, all carcinogenic events can be conveniently studied in the buccal mucosa which is easily accessible for cytologic smear preparation and does not cause undue stress to study subjects. ${ }^{[15]}$ Furthermore, more surface area of the mucosa is exposed to the insults in the oral cavity, making it more vulnerable to changes. ${ }^{[14]}$

Among DNA specific stains, for MN studies, most widely used are Feulgen and Acridine orange. Other nonspecific stains such as Giemsa, MayGrünwald-Giemsa (MGG), Papanicolaou and less frequently orcein, and hematoxylin and eosin were also used. ${ }^{[9]}$

According to observations made by Nersesyan et al. (2006) and Casartelli et al. (2000) micronuclei formation in epithelial cells may be overestimated when non-DNA specific stains like Giemsa stains are used. ${ }^{[16,17]}$ According to Thomas et al. (2009), Romanowsky stains (like MGG) have been shown to increase the number of false positives, as they positively stain keratin bodies that are often mistaken for micronuclei. Hence, they recommended that Feulgen stain be used because permanent slides can be obtained that can be viewed under both transmitted and/or fluorescent light conditions. ${ }^{[15]}$

Feulgen staining technique being highly DNA specific and also giving cytoplasm clear transparent appearance enables easy and distinct appearance of MN. Hence, the present study used Feulgen staining technique and binocular microscope for analysis of micronuclei which was simple and economical.

In the present study, MN frequencies were evaluated in controls as well as in PMDs. The levels of MN increased in PMDs as compared to that in controls. This finding correlated to that of studies done by Grover et al. (2012), Dindgire et al. (2012), and Sivasankari et al. (2008). ${ }^{[9,18,19]}$ Dindgire 
et al. (2012) and Sivasankari et al. (2008) included PMDs such as submucous fibrosis, leukoplakia, erythroplakia, and oral lichen planus in their respective studies whereas present study included all other groups except that of erythroplakia. ${ }^{[18,19]}$

Among subjects of three groups (except that of erythroplakia), in our study MN\% was highest in OSMF subjects followed by leukoplakia and then lichen planus. The findings of Sivasankari et al. (2008) were similar to that in our study where MN\% was highest in SMF patients followed by leukoplakia and lichen planus, whereas in a study done by Dindgire et al. (2012) MN\% was found to be highest in OSMF patients followed by lichen planus and leukoplakia cases. ${ }^{[18,19]}$

The levels of mean MN in PMDs such as leukoplakia, OSMF, and lichen planus compared to that of controls show a statistically significant difference in various studiesdone by Grover et al., Koneru and Kaveri, Jyoti et al., Mahimkar et al., and Buajeeb et al. ${ }^{[8,9,20-22]}$ According to Samanta and Dey (2010), the various possible explanations for $\mathrm{MN}$ formation in these lesions include chromosomal aberrations, chromosome loss/breakage, mitotic apparatus dysfunctions, aneuploidy, and genetic instability ${ }^{[4]}$ Variations in MN counts in different studies can be attributed to ingredients in the quid, the number of quids per day and the different lifestyles and food habits of people as these studies were conducted in different populations. ${ }^{[14]}$

On comparing occurrence of MN in OSMF and leukoplakia, in the present study no significant difference was found but on comparing occurrence of $\mathrm{MN}$ in lichen planus and leukoplakia a statistically significant difference was found. Contradictory to this Dindgire et al. (2012) did find a significant difference in mean $\mathrm{MN}$ occurrence between leukoplakia and OSMF. ${ }^{[18]}$ Hence, these findings of occurrence of MN in PMDs need further verification.

Dysplasia comprises a loss in the uniformity of the individual cells, as well as a loss in their architectural orientation. Furthermore, oral potentially malignant lesions with epithelial dysplasia are statistically more likely to progress to cancer. At present, the grade of dysplasia is regarded as the best guide to potential progression to OSCC. ${ }^{[23]}$ Hence, in the present study an attempt was made to correlate increase in grade of dysplasia with occurrence of micronuclei in exfoliated cells of buccal mucosa. The present study shows stepwise increase in MN frequency from stage of no dysplasia to stage of severe dysplasia. The study of Bhavsar et al. (2011) also had a similar finding of increased MN\% from stage of no dysplasia to severe dysplasia, like in the present study, it increased from $0.2 \%$ to $1.3 \%$ to $2.1 \%$. ${ }^{[7]}$

The findings in the present study and that in the study done by Bhavsar et al. (2011) has shown that MN are indicators of genotoxic damage and they do increase in number with increase in grade of dysplasia. ${ }^{[7]}$

Finally, in the present study, the main goal was to calculate and analyze frequency of micronuclei occurrence in normal subjects, i.e., without any harmful addictive habits (controls) and subjects with PMDs.

Increase in MN\% was observed from controls to PMD subjects in our study. These observations indicate cytogenetic damage in oral epithelium increase with increase in severity of disease. Kamboj and Mahajan (2007) also conducted a similar study and their findings using Feulgen stain reaction were $0.45 \%$ in controls, $1.70 \%$ in PMDs. ${ }^{[14]}$ It is evident that our findings are comparable to that of Kamboj and Mahajan (2007).

Similar findings were observed by Halder et al. (2004), Saran et al. (2008), Devi et al. (2011), Bhavsar et al. (2011), and Dindgire et al. (2012) in their respective studies. ${ }^{[7,10,15,24,25]}$

To summarize the results, assessment of the frequency of MN in oral exfoliated cells is an innovative technique, which holds promise for monitoring the genetic damage in PMDs.

\section{CONCLUSION}

The study concludes that the occurrence of $\mathrm{MN}$ and $\mathrm{MN} \%$ increases from controls to potentially malignant disorder cases. There is a statistically significant difference $(P<0.001)$ for occurrence of MN between controls and OPMD cases. Increase in occurrence of $\mathrm{MN}$ and $\mathrm{MN} \%$ was highest in patients of OSMF followed by leukoplakia and lichen planus patients. Last but not the least, with increase in grades of dysplasia, in OPMD subjects, the formation of MN increases.

Hence, it can be inferred from our study that the evaluation of $\mathrm{MN}$ frequencies in exfoliated buccal cells of PMDs using Feulgen stain can be utilized as a screening tool for early diagnosis and prevention of the disease as well as a way to reduce the mortality associated with the disease. 


\section{ACKNOWLEDGMENT}

Staff of Department of Oral Pathology and Department of Oral Medicine, Karnavati School of Dentistry, Uvarsad, Gandhinagar. Dr. Girish Mishra (MS ENT, Prof. and Guide, Department of Otolaryngology and Head and Necksurgery, Pramukhswami Medical College, Karamsad. Dr. Daxa Mishra MPT, Ph.D., K.M. Patel Institute of Physiotherapy, Karamsad.

\section{REFERENCES}

1. Uma AN, Dhananjay SK, Aroul T, Bakshi SS, Lokeshmaran A. Comparative cytogenetic study of exfoliative oral mucosal cells in tobacco related potentially malignant disorders in a South Indian population. Intl J Adv Res Tech 2014;3:6-12.

2. Kumar S, Debnath N, Ismail MB, Kumar A, Kumar A, Badiyani BK, et al. Prevalence and Risk factors for ora potentially malignant disorders in Indian population. Adv Prev Med 2015;2015:208519.

3. Palve DH, Tupkari JV. Clinico-pathological correlation of micronuclei in oral squamous cell carcinoma by exfoliative cytology. J Oral Maxillofac Pathol 2008;12:2-7.

4. Samanta S, Dey P. Micronucleus and its applications. Diagn Cytopathol 2012;40:84-90.

5. Akanksha G, Thimmarasa BV, Prashant J, Ankit G, Manas G, Kriti S. Micronuclei frequency as an early diagnostic tool for detection of Oral cancer: A comparative study. Intl J Oral Health Dent 2016;2:77-83.

6. Sabharwal R, Verma P, Syed MA, Sharma T, Subudhi SK, Mohanty S, et al. Emergence of micronuclei as a genomic biomarker. Indian J Med Paediatr Oncol 2015;36:212-8.

7. Bhavsar RS, Kumar GS, Hazarey VK, Sindhu GM. Cytomorphometric analysis for evaluation of cell diameter, nuclear diameter and micronuclei for detection of oral premalignant and malignant lesion. J Oral Biosci 2011;53:158-69.

8. Koneru A, Kaveri H. Comparative study of oral micronucleated cell frequency in oral submucous fibrosis patients and healthy individuals. J Clin Exp Dent 2011;3:201-6.

9. Grover S, Abr M, Jahagirdar A, Telagi N, Kulkarni PG. Comparative study for selectivity of micronuclei in oral exfoliated epithelial cells. J Cytol 2012;29:230-5.

10. Devi P, Thimmarasa VB, Mehrotra V, Arora P. Micronucleus assay for evaluation of genotoxicity in potentially malignant and malignant disorders. J Indian Acad Oral Med Radiol 2011;23:97-100.

11. Somashekhar P, Kamath VV. Evaluation of micronuclei in betel quid chewers, potentially malignant oral disorders and oral squamous cell carcinoma patients: A cytological assay. J Adv Clin Res Insights 2016;3:139-42.
12. Holland N, Bolognesi C, Kirsch-Volders M, Bonassi S, Zeiger E, Knasmueller $\mathrm{S}$, et al. The micronucleus assay in human buccal cells as a tool for biomonitoring DNA damage: The HUMN project perspective on current status and knowledge gaps. Mutat Res 2008;659:93-108.

13. Jadhav K, Gupta N, Ahmed MB. Micronuclei: An essential biomarker in oral exfoliated cells for grading of oral squamous cell carcinoma. J Cytol 2011;28:7-12.

14. Kamboj M, Mahajan S. Micronucleus an upcoming marker of genotoxic damage. Clin Oral Investig 2007;11:121-6.

15. Thomas P, Holland N, Bolognesi C, Kirsch-Volders M, Bonassi S, Zeiger E, et al. Buccal micronucleus cytome assay. Nat Protoc 2009;4:825-37.

16. Nersesyan A, Kundi M, Atefie K, Schulte-Hermann R, Knasmüller S. Effect of staining procedures on the results of micronucleus assays with exfoliated oral mucosa cells. Cancer Epidemiol Biomarkers Prev 2006;15:1835-40.

17. Casartelli G, Bonatti S, De Ferrari M, Scala M, Mereu P, Margarino G, et al. Micronucleus frequencies in exfoliated buccal cells in normal mucosa, precancerous lesions and squamous cell carcinoma. Anal Quant Cytol Histol 2000;22:486-92.

18. Dindgire SL, Gosavi S, Kumavat RM, Ganvir S, Hazarey V. Comparative study of exfoliated oral mucosal cell micronucleus frequency in potentially malignant and malignant lesions. Intl $\mathrm{J}$ Oral Maxillofacial Path 2012;3:15-20.

19. Sivasankari PN, Kaur S, Reddy KS, Vivekanandam S, Rao KR. Micronucleus index: An early diagnosis in oral carcinoma. J Anat Soc India 2008;57:8-13.

20. Jyoti S, Khan S, Afzal M, Naz F, Y SH. Evaluation of micronucleus frequency by acridine orange fluorescent staining in bucccal epithelial cells of oral submucosus fibrosis (OSMF) patients. Egypt $\mathrm{J}$ Med Hum Genet 2013;14:189-93.

21. Mahimkar MB, Samant TA, Kannan S, Patil T. Influence of genetic polymorphisms on frequency of micronucleated buccal epithelial cells in leukoplakia patients. Oral Oncol 2010;46:761-6

22. Buajeeb W, Kraivaphan P, Amornchat C, Triratana T. Frequency of micronucleated exfoliated cells in oral lichen planus. Mutat Res 2007;627:191-6.

23. Speight PM. Update on oral epithelial dysplasia and progression to cancer. Head Neck Pathol 2007;1:61-6.

24. Halder A, Chakraborty T, Mandal K, Gure PK, Das S. Comparative study of exfoliated oral mucosal cell micronuclei frequency in normal, precancerous and malignant epithelium. Int J Hum Genet 2004;4:257-60.

25. Saran R, Tiwari RK, Reddy PP, Ahuja YR. Risk assessment of oral cancer in patients with pre-cancerous states of the oral cavity using micronucleus test and challenge assay. Oral Oncol 2008;44:354-60. 